\title{
Oxidized ATM Protein Kinase Promotes the Proliferation of Breast CAF Through Upregulating the SCD1 Gene Expression by Activating Core Proliferation-Related Pathways
}

\section{Li Xing}

GuangXi University of Chinese Medicine

\section{Mingjun Ren}

GuangXi University of Chinese Medicine

\section{Wanying Bi}

GuangXi University of Chinese Medicine

\section{Wanjun Wu}

The Third Affliated Hospital of Guangxi University of Chinese Medicine

\section{Gui Jiang}

The Third Affiliated Hospital of Guangxi University of Chinese Medicine

\section{Limin Li}

Liuzhou People's Hospital affiliated to Guangxi Medical University

\section{Yu Lu}

Liuzhou People's Hospital Affiliated to Guangxi Medical University

\section{Hongli Yu}

Liuzhou People's Hospital Affiliated to Guangxi Medical University

\section{Manran Liu}

Chongqing Medical University

\section{Xin Tu}

The Affiliated Tumor Hospital of Guangxi Medical University

\section{Shifu Tang (D524908210@qq.com )}

Liuzhou People's Hospital affiliated to Guangxi Medical University https://orcid.org/0000-0001-53565409

\section{Research Article}

Keywords: Breast cancer, CAF, Oxidized ATM, SCD1, Proliferation

Posted Date: September 3rd, 2021 
DOI: https://doi.org/10.21203/rs.3.rs-843881/v1

License: (c) (1) This work is licensed under a Creative Commons Attribution 4.0 International License. Read Full License 


\section{Abstract}

\section{Purpose}

Stearoyl-CoA desaturase 1 (SCD1) is a key rate-limiting enzyme involved in the biosynthesis of monounsaturated fatty acids, which mediates lipid metabolism and participates in the proliferation of cancer cells. As a redox sensor, ATM protein kinase is also involved in DNA repair and cell proliferation regulation. Previous studies have shown that oxidized ATM protein kinase can facilitates the proliferation of breast CAF via by regulating the cell glucose and protein metabolism. However, lipid metabolism is essential for cell proliferation. Whether the oxidized ATM protein kinase can regulate the expression of the SCD gene to promote the lipid metabolism is unknown.

\section{Methods}

We use immunohistochemical techniques to observe the level of SCD1 and oxidized ATM protein kinase, MTT Assay and flow cytometric analysis observes the proliferation of breast CAFs, and Western Blot techniques analyze the expression of SCD gene expression.

\section{Results}

The expression levels of SCD1 protein expression in breast cancer were closely related to oxidized ATM expression $(P<0.05)$. And SCD promotes the proliferation of breast CAF cells. The oxidized ATM protein kinase can promote the proliferation of breast CAFs by upregulation of SCD protein expression levels.

\section{Conclusion}

Our results indicate that oxidized ATM protein kinase may play a central role in promoting the proliferation of breast CAF by coordinating glucose, protein and lipid metabolism. oxidized ATM can facilitate the breast CAFs Lipid metabolism by upregulating the SCD1 gene expression.

\section{Background}

Breast cancer (BC) is one of the frequent malignant cancers in women around the world [1], which is the result of long-term interaction between breast cancer cells and tumor microenvironment[2]. In tumor microenvironment, cancer-associated fibroblasts (CAF) are the major stromal cells, which promote tumor growth, vascularization, invasion and metastasis [3]. Compared with normal fibroblasts (NF), CAF has an abnormal proliferation behavior, which is mediated by oxidative stress and cell key redox sensors such as ATM protein kinase [4, 5]. Ataxia telangiectasia mutated (ATM) protein kinase is encoded by ATM genes and belongs to the phosphatidylinositol 3 kinase-like protein kinase family,which is responsible for the ataxia-telangiectasia syndrome (A-T) [1, 6]. As a core redox sensor, ATM protein kinase can be directly activated by oxidative stress independent of double strand breaks in cytoplasm. In the cytoplasm, hydrogen peroxide oxidizes the mercaptan group on the 2991 cysteine residue of ATM to form a hyposulfonic group, which covalently combines with the mercaptan group on the 2991 cysteine residue 
of another ATM protein monomer to form an intermolecular disulfide bond, which transforms the inactive ATM dimer into an active ATM dimer[5]. Our previous work showed that oxidized ATM promotes abnormal proliferation of breast CAFs through maintaining intracellular redox homeostasis and activating the PI3K-AKT, MEK-ERK, and Wnt- $\beta$-catenin signaling pathways[4]. In recent years, oxidized ATM promotes cell proliferation by regulating cell metabolism[7]. Klising sireul et al. reported for the first time in $\mathbf{2 0 0 6}$ that under ion irradiation, genes related to cholesterol metabolism in ATM kinase deficient lymphocytes were down regulated, resulting in the decrease of cholesterol content and inhibition of cell proliferation, suggesting oxidized ATM may play an important role in controlling the proliferation by regulating lipid metabolism [8]. As a protein kinase, oxidized ATM likely regulates many unknown substrates and / or related signaling pathways, which are closely related to lipid metabolism, to regulate the proliferation of breast CAF, but it needs to be clarified.

Stearoyl CoA desaturase 1 (SCD1) is an endoplasmic reticulum integral membrane protein which belongs to the fatty acid desaturase family and is the key enzyme responsible for catalyzing the synthesis of mono-unsaturated fatty acids (MUFAs), mainly palmitoleate and oleate, from saturated fatty acids (SFAs), stearate and palmitate, respectively[9]. SFAs and MUFAs are the main components of mammalian cell lipids, source of energy and signal molecules, which are the basic components of biofilms [10]. To date, two human isoforms (1 and 5) have been identified. Human SCD1 is widely expressed in tissues, whereas human SCD5 is predominantly expressed in the brain and pancreas [11, 12]. SCD1 expression is obviously elevated in many kinds of human cancer [13]. SCD1 has been found to be an important regulator of cell proliferation. SCD facilitates the proliferation of prostate cancer cells through transactivating of androgen receptor [14]. SCD1 advances the proliferation of breast cancer cells and gastric cancer cells [15-17]. Existing studies have shown that PI3K / Akt and other important cell proliferation signaling pathways play an important role in regulating SCD1 gene expression. In 2007, Mauvoisin et al. found that insulin upregulates PI3K/mTOR-SREBP-1 signaling pathway and activates SCD1 gene expression in HepG2 hepatoma cells [18]. Subsequently, AKT kinase activation was found to up regulate SCD expression [19]. Our previous work has shown that PI3K-AKT and ERK 1/2 are regulated by oxidative ATM protein kinase in breast CAF[4]. However, it is unclear whether and how SCD1 mediate the role of oxidized ATM in promoting the proliferation of CAF from breast cancer.

This study illustrated that oxidized ATM protein kinase promotes the proliferation of breast CAF proliferation by activating crucial proliferation-related signaling pathways, which upregulate the protein expression of SCD1 gene. Function loss of oxidized ATM caused by KU60019, a specific ATM kinase inhibitor, downregulates the protein expression levels of SCD1 gene, so does the inactivation of the ERK, PI3K-AKT, and WNT cell proliferation regulatory pathways by the respective specific inhibitors. Inhibition of SCD1 gene expression by CAY 10566 obviously blocks the proliferation of breast CAF. The protein expression levels of SCD1 gene in CAF of breast cancer tissues are higher than that of NF of breast paracancer tissues, and closely correlated to the protein expression of oxidized ATM and the clinical staging of breast cancer. Therefore, the SCD1 protein mediates the proliferation of breast CAF regulated by the oxidative ATM / proliferation-related signal pathways axis. 


\section{Materials And Methods}

\section{Clinical data}

A total of 42 cases of breast cancer were collected from The Third Affiliated Hospital of Guangxi University of Chinese Medicine. The postoperative pathological specimens were confirmed. Normal breast tissues adjacent to the cancer were collected as a control. The clinicopathological data of all the enrolled patients were collected, including age, tumor size, lymph node metastasis, etc., and the clinical staging was conducted according to the TNM staging criteria of the International Union against Cancer in 2017.The clinical data of all patients also included the detection results of Estrogen receptor( ER), Progesterone receptor (PR) and Human epidermal growth factor receptor 2खHER-2】.This study was approved by the Ethics Committee of The Third Affiliated Hospital of Guangxi University of Chinese Medicine, and all patients signed an informed consent form.

\section{Immunohistochemistry (IHC) staining}

IHC was carried out as described previously[20]. The tumor specimens and its adjacent normal tissues were immobilized with formalin. Paraffin-embedded specimens were sectioned into $4 \mu \mathrm{M}$ sections and stained with H\&E according to standard histopathological techniques. After being treated with $3 \%$ hydrogen peroxide in methanol for 30 min to exhaust endogenous peroxidase activity, the slices were incubated with rabbit monoclonal antibody of ATM (2873, CST, 1:50 dilution), rabbit monoclonal p-ATM antibodies (s1981) (5883, CST, 1:50 dilution), rabbit polyclonal antibody of SCD1 (AF7944, Beyotime, 1:50 dilution) at $4{ }^{\circ} \mathrm{C}$ overnight. Then, the sections were sequentially incubated with polymer helper solution (ZSBiO) for 20 min, polyperoxidase-antimouse/rabbit IgG (PV-9000, ZSBiO, 1:100 dilution) for 30 min at $37 \mathrm{C}$, and stained with diaminobenizidine. All images (100x) were captured under a constant light source using a Nikon Eclipse 80i microscope.

\section{Cell lines and cell culture}

Immortalized paired NFs and CAFs cell lines derived from breast cancer were donated by Professor Manran Liu of Chongqing Medicine University. These cell lines were routinely cultured in full DMEM (41965-062, Gibco) supplemented with 10\% fetal bovine serum (100099-133, Gibco) and penicillin/streptomycin double-resistant fluid (15070-063, Gibco), cultured in moist air with $5 \% \mathrm{CO}_{2}$ at $37^{\circ} \mathrm{C}$.

\section{MTT assay and flow cytometric analysis}

Cell proliferation rate was measured by 3- (4,5-1, 3-dimethyl-2-thiazolium) -2, 5-diphenyl-2-h-tetrazolium bromide (MTT) method. Cells were inoculated to 96 -well plates at a cell density of $3 \times 10^{3}$ cells per well. When the fusion growth reached $60 \%$, different doses of CAY-10566 $(0,5,10,20 \mu \mathrm{M})$ were maintained for different days. Next, $10 \mathrm{ml}$ of MTT solution (C0009, Beyotime) $(5 \mathrm{mg} / \mathrm{ml}$, in PBS solution) were added to each well and incubated for 4 hours. Carefully remove the supernatant, add $200 \mathrm{ml}$ of DMSO to each well, 
and gently shake the rotator for 10 minutes in a dark environment at room temperature. The wavelength of $492 \mathrm{~nm}$ was selected to measure the absorption of each well on the ultraviolet spectrophotometer, and the results were recorded. The experiment was repeated three times.

The cell cycle distribution was analyzed by flow cytometry. Cell DNA staining was performed using the standard method of propidium iodide as described earlier[20]. Briefly, after treatment, the cells were trypsinized, washed twice with cold PBS, fixed by $75 \%$ ethanol, incubated with RNAase and stained by propidium iodide (P4170, Sigma Aldrich). Tritiated thymidine uptake of cells was determined using a cytomics FC device (Beckman coulter). The experiments were performed in triplicate.

\section{Cell growth density and cellular morphology}

The cells were inoculated in 6 -well plates at a density of $5 \times 10^{5}$ cells/well. $2 \mathrm{~mL}$ DMEM culture medium was added to each well and the cells were cultured. When the fusion growth reached $60 \%$, specific reagents were added in the subsequent treatment according to the experimental design, and the cell density and morphology were observed at the designed time points using a phase contrast microscope (Nikon, TE2000- V)

\section{Western blot analysis}

Cells were inoculated to 96 -well plates at a density of $3 \times 10^{3}$ cells per well. When $60 \%$ confluence was reached, the cells were treated with KU60019(S1570, Selleck chemical), LY294002(S1105, Selleck chemical), U0126(A1901, Beyotime), and XAV939(S1180, Selleck chemical) for $24 \mathrm{~h}$. Total cellular proteins were extracted from RIPA lysate (P0013B, Beyotime), quantitatively isolated on 10\% SDS-PAGE gel and imprinted on protein gel using BCA protein assay kit (P0012, Beyotime). The primary antibodies include polyclonal antibody of SCD1 rabbit (AF7944, Beyotime, 1:500 dilution), monoclonal mouse $\beta$ Actin antibody (AA128, Beyotime, 1:1000 dilution), monoclonal antibody of p21CIP1 in mice (AP021 Beyotime, 1:500 dilution), p- RB (s795) polyclonal rabbit antibody (BS6414, Bioworld, 1:500 dilution), c Myc rabbit monoclonal antibodies (5605, CST, the latter dilution). After incubating with a primary antibody at $4{ }^{\circ} \mathrm{C}$ overnight. Appropriate horseradish peroxidase (HRP) labeled secondary antibody (Beyotime) was added, followed by immunoassay using an enhanced chemiluminescence system (CoolImager). All experiments were repeated at least 3 times.

\section{Statistical analysis}

SPSS 13.0 software was used for statistical analysis. Repeat each experiment at least three times. The absorbance values of CAF with the CAY10566 treatment were expressed as mean \pm standard deviation (mean \pm SD) for $t$ test. The count data was expressed in numbers or (\%). The differential expression of ATM, P-ATM and SCD1 in breast cancer tissues and paracancer tissues, as well as the correlationship between SCD1 expression and clinicopathology parameters in breast cancer patients, were analyzed by Pearson's chi-square test and Fisher's exact probability method. Spearman method was used to analyze 
the correlationship between p-ATM (s1981) and SCD1 expression. $P<0.05$ was considered statistically significant.

\section{Results}

\section{SCD1 protein expression levels are closely related to oxidized ATM protein expression levels in CAF of breast cancer tissue}

To shed light on the correlation between SCD1 and oxidative ATM protein expression in CAF from breast cancer, we collected breast cancer tissues and paracancer tissues as well as their corresponding pathological data and used immunohistochemical methods to detect the protein expression levels of ATM, p- ATM(s1981) and SCD1 in CAF of breast cancer. As can be seen in table 1- table 3 and figure 1 , the protein expression levels of ATM, P-ATM and SCD1 of CAF all exceed that of NF in breast cancer $(P<$ 0.05). Moreover, in the cell lines of immortalized breast cancer NF/CAF, the protein expression levels of CAF are higher than that of NF from breast cancer $(P<0.05)$ (supplementary figure 1 ), which was consistent with the results of SCD1 immunohistochemistry staining in breast cancer (table 3). As expected, there is a positive correlation between SCD1 protein expression levels and p-ATM(s1981) protein expression levels in CAF from breast cancer $(P<0.05)$ (Table 4). It was also shown that SCD1 protein expression levels were not significantly correlated with gender, age, tumor size, lymph node metastasis, distant metastasis, pathological grade, PR, ER and HER-2 ( $P \bowtie 0.05)$, except for TNM stage $(P<$ 0.05) (Table 5). The above evidence demonstrated that SCD1 protein expression levels are closely related to that of oxidized ATM in CAF from breast cancer, suggesting oxidized ATM protein kinase may control the proliferation of CAF via SCD1.

\section{SCD1 promotes the proliferation of CAF in breast cancer}

To explore the role of SCD1 in the abnormal proliferation of CAFs, we treated CAF cells with the SCD1 specific inhibitor CAY-10566 and then observed the cell growth curve, growth density, cell cycle distribution and proliferation-associated key protein regulators of CAF. As shown in figure 2, cell growth rate, growth density and cell proportion in S phase are reduced obviously after the CAY-10566 treatment, implying SCD1 plays an essential role in the proliferation of breast cancer CAF. What is more, under the condition of CAY-10566 treatment, the protein expression levels of p-RB and c-Myc decreased, but that of P21 increased (Figure 2D), suggesting SCD1 promotes the proliferation of breast cancer CAF.

\section{Oxidative ATM protein kinase promotes the proliferation of breast cancer CAF via upregulating the protein expression of SCD1}

Our previous research showed that several core proliferations signaling pathways including PI3K-AKT, ERK and WNT are regulated by oxidized ATM in breast cancer CAF, while SCD1 is located downstream of these signaling pathways $[4,18]$. In order to determine whether oxidized ATM controls the proliferation of breast cancer CAF through regulating the expression of SCD1, we tested the protein expression levels of SCD1 in breast cancer CAF with or without the treatment of CAY-10566, KU60019(ATM inhibitor), 
LY294002 (PI3K inhibitor), U0126 (MEK/ERK inhibitor), or XAV939 ( $\beta$-catenin inhibitor). As shown in Figure 3A, CAY- 10566 can inhibit the protein expression of SCD1. SCD1 protein expression in breast cancer CAF was also inhibited by the KU60019 treatment in dose or time-dependent manner (Figure 3B, 3C and 3D). Consistent with this result, LY294002, U0126, or XAV939 alone inhibited the protein expression of SCD1 in CAF (Figure 3E). These above results indicate that oxidized ATM promotes the proliferation of breast cancer CAF via upregulating the expression of SCD1 through activating the PI3KAKT, ERK and WNT signaling pathways.

\section{Discussion}

It's well established that CAF is characterized with abnormal proliferation[21, 22], but the underlying mechanisms are not completely clarified[23]. Our previous work has shown that oxidized ATM protein kinase facilitates the proliferation of breast CAF via maintaining intracellular redox homeostasis and activating the PI3K-AKT, ERK-MAPK, and Wnt signaling pathways[4]. Several studies have reported that oxidized ATM not only regulates the cell glucose and protein metabolism [24,25], but also is involved in the cell lipid metabolism [8, 26]. Lipid metabolism is indispensable for cell proliferation[9]. Whether oxidized ATM promotes the breast CAF proliferation through regulating the cell lipid metabolism is unknown. In this study, oxidized ATM was demonstrated to facilitate the proliferation of breast CAF via increasing the SCD1 gene expression by activating PI3K, ERK, and Wnt signaling pathways. To our best knowledge, it is first reported that oxidized ATM protein kinase promotes the proliferation of breast CAF via regulating lipid metabolism through activating the core proliferation signaling pathways/ SCD1 axis.

Oxidized ATM facilitates the proliferation of breast CAF partially by enhancing lipid anabolism through enhancing the SCD1 gene expression. Insulin resistance and growth impairments are the peculiar characteristics of ataxia-telangiectasia patients [27, 28], indicating oxidized ATM is necessary for glucose metabolism and cell proliferation. Oxidized ATM is required for AKT phosphorylation at Ser473 to fully activate the PI3K/AKT pathway and for membrane translocation of the cell-surface glucose transporter 4 in response to insulin treatment [29,30], suggesting oxidized ATM can enhance the cell proliferation by boosting cell glucose absorption. Our previous studies have shown that oxidized ATM promotes the proliferation of breast CAF not only by activating the key proliferation signaling pathways but also by increasing cell glucose metabolism $[4,25]$. So, it's reasonable to infer that oxidized ATM could facilitate the breast CAF proliferation by regulating cell lipid metabolism. Many previous studies showed that SCD1-mediated lipid metabolism has been the main focus in the field of cell proliferation-relevant study. SCD1 is a central rate-limiting lipogenic enzyme and catalyzes the synthesis of MUFAs, primarily oleate and palmitoleate[9]. These MUFAs are further synthesized into different lipid species such as phospholipids, cholesteryl esters, and triglycerides, which are required for cell proliferation[18]. The expression of the SCD1gene is often reported to be increased and promote cell proliferation in many cancers $[13,31]$. Klising sireul et al. reported in 2006 ATM kinase insufficiency-caused cholesterol metabolism defect inhibit cell proliferation[8]. Whether oxidized ATM promotes the breast CAF 
proliferation via regulating the SCD1 gene expression remains to be investigated. In this current work, the protein expression levels of SCD1 in CAF were higher than that of NF in breast cancer (Fig. 1); inhibition of oxidized ATM by KU60019 or of key proliferation pathways by their inhibitors significantly suppressed the SCD1 gene expression in breast CAF (Fig. 3B-3D) and the inhibition of the SCD1 gene expression by its special inhibitor CAY10566 obviously blocked the breast CAF proliferation (Fig. 2 and Fig. 3A). As far as we know, it is the first time reported that oxidized ATM enhances the proliferation of breast CAF via upregulating the SCD1 gene expression. It's noteworthy that oxidized ATM directly phosphorylates elF-4Ebinding protein 1(4E-BP1) at Ser 111 to initiate cell protein synthesis, which is a prelude to cell growth and division[32]. The above evidence implies that oxidized ATM protein kinase perhaps plays a central role in promoting breast CAF proliferation through coordinating glucose, protein, and lipid metabolism.

SCD1 partly mediates the role of oxidized ATM in promoting the breast CAF proliferation. Hitherto, it's not reported that how oxidized ATM regulates the SCD1 gene expression. However, accumulating evidence suggests that oxidized ATM may indirectly regulate the SCD1 gene expression in breast CAF. First, the SCD1 gene expression is activated by the PI3K/mTOR/SREBP-1 axis in response to insulin stimulus in liver[18]. Secondly, SCD1 is essential for the activation of PI3K-mTOR/AKT and WNT signaling pathways and inactivation of AMPK pathway [33,34]. Activated mTOR can promote the transcription of SREBP-1 mRNA and the processing of SREBP-1, which in turn induces the expression of fat, including ACLY, ACC, FASN and SCD1. This proves that one of the main mechanisms by which AKT/mTOR promotes tumorigenesis is the promotion of cancer metabolism, especially fat generation[16-18, 35]. Lastly, the PI3K-mTOR/AKT and WNT proliferation signaling pathways are activated in breast CAF[4]. However, how oxidized ATM promotes the abnormal proliferation of breast CAFs in a SCD1 dependent manner by regulating the activity of these pathways remains to be investigated. In this work, we found that inhibition of the PI3K, ERK, and WNT signaling pathways by respective specific inhibitor reduces the SCD1 gene expression in breast CAF [figure 3E], indicating that oxidized ATM can facilitate the breast CAFs proliferation by upregulating the SCD1 gene expression through activating the PI3K-AKT/mTOR, ERK, and WNT signaling pathways. Nevertheless, the underlying mechanisms of who oxidized ATM/ ERK or WNT signaling axis upregulates the SCD1gene expression needs to be investigated.

Post-translational modification is very important for functional proteins to perform their functions by modulating these substrate activity, stability, subcellular localization and interaction with other proteins. Phosphorylation modification can fine-tune the function of a substrate. Oxidized ATM protein kinase not only promotes the breast CAF proliferation via activating key proliferation signaling pathways through phosphorylating the core members of these pathways, but also accelerating glucose absorption via increasing membrane translocation of the human glucose transporter 1(GLUT 1) through directly phosphorylating GLUT1 at S490 [4, 25]. It's possible that versatile oxidized ATM protein kinase may promote the proliferation of breast CAF by phosphorylating key proteins involved in lipid metabolism, but remains to be explored in the future.

In short, this study shows that oxidized ATM facilitates breast CAFs proliferation via upregulating the SCD1gene expression through activating core proliferation signaling pathways. These findings provide 
novel targets for treating breast cancer.

\section{Declarations}

\section{Acknowledgements}

This work was supported in part by National Natural Science Foundation of China. (NSFC815604308X

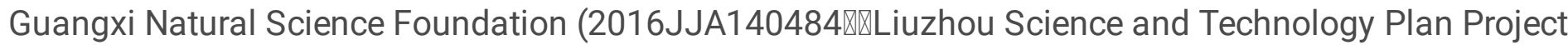
(2020NBAB0827), The Scientific Research Project of Guangxi Health and Family Planning Commission (Z20170666), Innovation Project of Guangxi Graduate Education (YCSZ2020026)『YCSZ2020014】

Conflicts of Interest.

We have no conflicts of interest.

\section{References}

1. Prokopcova J, Kleibl Z, Banwell CM, Pohlreich P: The role of ATM in breast cancer development. Breast cancer research and treatment 2007, 104(2):121-128.

2. Ben-Baruch A: Host microenvironment in breast cancer development: inflammatory cells, cytokines and chemokines in breast cancer progression: reciprocal tumor-microenvironment interactions. Breast cancer research : BCR 2003, 5(1):31-36.

3. Houthuijzen JM, Jonkers J: Cancer-associated fibroblasts as key regulators of the breast cancer tumor microenvironment. Cancer metastasis reviews 2018, 37(4):577-597.

4. Tang S, Hou Y, Zhang H, Tu G, Yang L, Sun Y, Lang L, Tang X, Du YE, Zhou M et al: Oxidized ATM promotes abnormal proliferation of breast CAFs through maintaining intracellular redox homeostasis and activating the PI3K-AKT, MEK-ERK, and Wnt- $\beta$-catenin signaling pathways. Cell cycle (Georgetown, Tex) 2015, 14(12):1908-1924.

5. Guo Z, Kozlov S, Lavin MF, Person MD, Paull TT: ATM activation by oxidative stress. Science 2010, 330(6003):517-521.

6. Antonelli M, Strappazzon F, Arisi I, Brandi R, D'Onofrio M, Sambucci M, Manic G, Vitale I, Barilà D, Stagni V: ATM kinase sustains breast cancer stem-like cells by promoting ATG4C expression and autophagy. Oncotarget 2017, 8(13):21692-21709.

7. S.Tang et al., Research progress of the role of ATM protein kinase promotes cell proliferation. Molecular diagnosis and treatment, 2020, 249-252+258.

8. Klising-Sireul E, Rigaud O, Ory K, Ugolin N, Lebeau J, Levalois C, Lectard B, Chevillard S: Transcriptional response of wild-type and ataxia telangiectasia lymphoblasts following exposure to equitoxic doses of ionizing radiation. Journal of radiation research 2006, 47(3-4):259-272.

9. AM AL, Syed DN, Ntambi JM: Insights into Stearoyl-CoA Desaturase-1 Regulation of Systemic Metabolism. Trends in endocrinology and metabolism: TEM 2017, 28(12):831-842. 
10. Angelucci C, Maulucci G, Colabianchi A, lacopino F, D'Alessio A, Maiorana A, Palmieri V, Papi M, De Spirito M, Di Leone A et al: Stearoyl-CoA desaturase 1 and paracrine diffusible signals have a major role in the promotion of breast cancer cell migration induced by cancer-associated fibroblasts. British journal of cancer 2015, 112(10):1675-1686.

11. Castro LF, Wilson JM, Gonçalves O, Galante-Oliveira S, Rocha E, Cunha I: The evolutionary history of the stearoyl-CoA desaturase gene family in vertebrates. BMC evolutionary biology 2011, 11:132.

12. Wang J, Yu L, Schmidt RE, Su C, Huang X, Gould K, Cao G: Characterization of HSCD5, a novel human stearoyl-CoA desaturase unique to primates. Biochemical and biophysical research communications 2005, 332(3):735-742.

13. Tracz-Gaszewska Z, Dobrzyn P: Stearoyl-CoA Desaturase 1 as a Therapeutic Target for the Treatment of Cancer. Cancers 2019, 11(7).

14. Kim SJ, Choi H, Park SS, Chang C, Kim E: Stearoyl CoA desaturase (SCD) facilitates proliferation of prostate cancer cells through enhancement of androgen receptor transactivation. Molecules and cells 2011, 31(4):371-377.

15. Wang C, Shi M, Ji J, Cai Q, Zhao Q, Jiang J, Liu J, Zhang H, Zhu Z, Zhang J: Stearoyl-CoA desaturase 1 (SCD1) facilitates the growth and anti-ferroptosis of gastric cancer cells and predicts poor prognosis of gastric cancer. Aging 2020, 12(15):15374-15391.

16. Zhao J, Zhang LT, Bai Y, Wang ZY, Zhang XM, Ma CL: [Inhibition of SCD1 Activity Blocks Cell Cycle Progression and Impairs Proliferation in Breast Cancer Cells]. Sichuan da xue xue bao Yi xue ban = Journal of Sichuan University Medical science edition 2019, 50(4):546-550.

17. Luyimbazi D, Akcakanat A, McAuliffe PF, Zhang L, Singh G, Gonzalez-Angulo AM, Chen H, Do KA, Zheng $\mathrm{Y}$, Hung MC et al: Rapamycin regulates stearoyl CoA desaturase 1 expression in breast cancer. Molecular cancer therapeutics 2010, 9(10):2770-2784.

18. Mauvoisin D, Rocque G, Arfa O, Radenne A, Boissier P, Mounier C: Role of the PI3-kinase/mTor pathway in the regulation of the stearoyl CoA desaturase (SCD1) gene expression by insulin in liver. Journal of cell communication and signaling 2007, 1(2):113-125.

19. Li L, Wang C, Calvisi DF, Evert M, Pilo MG, Jiang L, Yuneva M, Chen X: SCD1 Expression is dispensable for hepatocarcinogenesis induced by AKT and Ras oncogenes in mice. PloS one 2013, 8(9):e75104.

20. Zhang H, Hou Y, Xu L, Zeng Z, Wen S, Du YE, Sun K, Yin J, Lang L, Tang X et al: Cytoplasmic Drosha Is Aberrant in Precancerous Lesions of Gastric Carcinoma and Its Loss Predicts Worse Outcome for Gastric Cancer Patients. Digestive diseases and sciences 2016, 61(4):1080-1090.

21. Peng Q, Zhao L, Hou Y, Sun Y, Wang L, Luo H, Peng H, Liu M: Biological characteristics and genetic heterogeneity between carcinoma-associated fibroblasts and their paired normal fibroblasts in human breast cancer. PloS one 2013, 8(4):e60321.

22. Wang L, Hou Y, Sun Y, Zhao L, Tang X, Hu P, Yang J, Zeng Z, Yang G, Cui X et al: c-Ski activates cancer-associated fibroblasts to regulate breast cancer cell invasion. Molecular oncology 2013, 7(6):1116-1128. 
23. Tang S, Yang L, Tang X, Liu M: The role of oxidized ATM in the regulation of oxidative stress-induced energy metabolism reprogramming of CAFs. Cancer letters 2014, 353(2):133-144.

24. Ditch S, Paull TT: The ATM protein kinase and cellular redox signaling: beyond the DNA damage response. Trends in biochemical sciences 2012, 37(1):15-22.

25. Sun K, Tang S, Hou Y, Xi L, Chen Y, Yin J, Peng M, Zhao M, Cui X, Liu M: Oxidized ATM-mediated glycolysis enhancement in breast cancer-associated fibroblasts contributes to tumor invasion through lactate as metabolic coupling. EBioMedicine 2019, 41:370-383.

26. Zhang YJ, Zhao H, Dong L, Zhen YF, Xing HY, Ma HJ, Song GY: Resveratrol ameliorates high-fat dietinduced insulin resistance and fatty acid oxidation via ATM-AMPK axis in skeletal muscle. European review for medical and pharmacological sciences 2019, 23(20):9117-9125.

27. Bar RS, Levis WR, Rechler MM, Harrison LC, Siebert C, Podskalny J, Roth J, Muggeo M: Extreme insulin resistance in ataxia telangiectasia: defect in affinity of insulin receptors. The New England journal of medicine 1978, 298(21):1164-1171.

28. Schalch DS, McFarlin DE, Barlow MH: An unusual form of diabetes mellitus in ataxia telangiectasia. The New England journal of medicine 1970, 282(25):1396-1402.

29. Viniegra JG, Martínez N, Modirassari P, Hernández Losa J, Parada Cobo C, Sánchez-Arévalo Lobo VJ, Aceves Luquero Cl, Alvarez-Vallina L, Ramón y Cajal S, Rojas JM et al: Full activation of PKB/Akt in response to insulin or ionizing radiation is mediated through ATM. The Journal of biological chemistry 2005, 280(6):4029-4036.

30. Halaby MJ, Hibma JC, He J, Yang DQ: ATM protein kinase mediates full activation of Akt and regulates glucose transporter 4 translocation by insulin in muscle cells. Cell Signa/ 2008, 20(8):15551563.

31. Peck B, Schug ZT, Zhang Q, Dankworth B, Jones DT, Smethurst E, Patel R, Mason S, Jiang M, Saunders $\mathrm{R}$ et al: Inhibition of fatty acid desaturation is detrimental to cancer cell survival in metabolically compromised environments. Cancer \& metabolism 2016, 4:6.

32. Yang DQ, Kastan MB: Participation of ATM in insulin signalling through phosphorylation of elF-4Ebinding protein 1. Nature cell biology 2000, 2(12):893-898.

33. Tan SH, Shui G, Zhou J, Shi Y, Huang J, Xia D, Wenk MR, Shen HM: Critical role of SCD1 in autophagy regulation via lipogenesis and lipid rafts-coupled AKT-FOX01 signaling pathway. Autophagy 2014, 10(2):226-242.

34. Igal RA: Stearoyl-CoA desaturase-1: a novel key player in the mechanisms of cell proliferation, programmed cell death and transformation to cancer. Carcinogenesis 2010, 31(9):1509-1515.

35. Igal RA: Roles of StearoylCoA Desaturase-1 in the Regulation of Cancer Cell Growth, Survival and Tumorigenesis. Cancers 2011, 3(2):2462-2477.

\section{Tables}


Table 1. The comparision of total ATM protein expression between breast cancer tissues and adjacent non-tumor breast tissues

\begin{tabular}{|c|c|c|c|}
\hline \multicolumn{4}{|c|}{ Total ATM protein expression } \\
\hline & Negative $\rrbracket n, \% \rrbracket$ & Positive $\rrbracket n, \% \bigotimes$ & $P$-value \\
\hline Breast cancer tissues & $9 \rrbracket 21.43 \rrbracket$ & $33 \rrbracket 78.57 \rrbracket$ & 0.00 \\
\hline Peritumoral breast tissues & $32 \bigotimes 76.19 \rrbracket$ & $10 \rrbracket 23.81 \rrbracket$ & \\
\hline
\end{tabular}

Table 2. The comparision of p-ATM(s1981) protein expression in breast cancer tissues and adjacent nontumor breast tissues

\begin{tabular}{|c|c|c|c|}
\hline \multicolumn{4}{|c|}{ p-ATM(s1981) protein expression } \\
\hline & Negative $₫ n, \% \rrbracket$ & Positive $\mathbb{n}$, \%】 & $P$-value \\
\hline Breast cancer tissues & $4 \rrbracket 9.52 \rrbracket$ & 38ه90.48囚 & 0.00 \\
\hline Peritumoral breast tissues & $33 \llbracket 78.57 \rrbracket$ & $9 \rrbracket 21.43 \rrbracket$ & \\
\hline
\end{tabular}

Table 3. The comparision of SCD1 protein expression in breast cancer tissues and adjacent non-tumor breast tissues

\begin{tabular}{|c|c|c|c|}
\hline & \multicolumn{3}{|c|}{ SCD1 protein expression } \\
\hline & Negative $₫ n, \% \rrbracket$ & 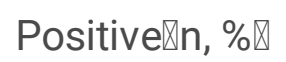 & $P$-value \\
\hline Breast cancer tissues & 8\19.05》 & $34 \llbracket 80.95 \rrbracket$ & 0.00 \\
\hline Peritumoral breast tissues & $33 \bigotimes 78.57 \rrbracket$ & $9 \rrbracket 21.43 \rrbracket$ & \\
\hline
\end{tabular}

Table 4. The pearson correlation analysis of SCD1 and p-ATM(s1981) expression in CAF of breast cancer tissues

\begin{tabular}{|c|c|c|c|}
\hline \multirow{2}{*}{$\begin{array}{c}\text { p-ATM(s1981) } \\
\text { expression }\end{array}$} & \multicolumn{2}{|c|}{ SCD1 expression } & \multirow[b]{2}{*}{$P$-value } \\
\hline & Positive $\ n, \% \rrbracket$ & Negative $\rrbracket n, \% \bigotimes$ & \\
\hline Positive $\ n, \% \bigotimes$ & $33 \otimes 78.57 \rrbracket$ & $5 \rrbracket 11.90 \rrbracket$ & 0.003 \\
\hline Negative $\rrbracket n, \% \rrbracket$ & $1 \rrbracket 2.38 \rrbracket$ & $3 \otimes 7.14 \rrbracket$ & \\
\hline
\end{tabular}


Table 5. The correlation analysis of SCD1 protein expression and clinical pathological characteristics of breast cancer tissues 
Pathological parameters

Number SCD negative SCD1 positive

$\begin{array}{llll}\mathrm{n} & \% & \mathrm{n} & \%\end{array}$

Gender

male

female

41

$1 \quad 0$

$\begin{array}{ll}0 & 0.00\end{array}$

$1 \quad 100.00$

Age(years)

$\leq 55$

24

$6 \quad 25.00$

$18 \quad 75.00$

$>55$

18

15.56

$17 \quad 94.44$

Tumor size $(\mathrm{cm})$

$\leq 5$

32

$9 \quad 28.13$

$23 \quad 71.87$

$>5$

10

$4 \quad 40.00$

$6 \quad 60.00$

Lymph node metastasis

$\begin{array}{llllll}\text { no } & 21 & 3 & 14.86 & 18 & 85.14 \\ \text { yes } & 21 & 5 & 23.81 & 16 & 76.19\end{array}$

Distant metastasis

\begin{tabular}{llllll} 
no & 37 & 6 & 16.22 & 31 & 83.78 \\
\hline yes & 5 & 2 & 40.00 & 3 & 60.00 \\
\hline Differentiation grading & & & & & \\
\hline low & 7 & 1 & 14.26 & 6 & 85.74 \\
middle & 24 & 4 & 16.67 & 20 & 83.33 \\
\hline high & 11 & 3 & 27.27 & 8 & 72.73
\end{tabular}

Staging ${ }^{a}$

$\begin{array}{llllll}\square+\square & 29 & 3 & 10.34 & 26 & 89.66 \\ \square+\square & 13 & 6 & 46.15 & 7 & 53.85\end{array}$

Progesterone receptor $\ \mathrm{PR} \rrbracket$

negative

positive

Estrogen receptorXER】

negative

$\begin{array}{lllll}16 & 3 & 18.75 & 13 & 81.25 \\ 26 & 5 & 19.23 & 21 & 80.77\end{array}$

$\begin{array}{lllll}15 & 4 & 26.67 & 11 & 73.33\end{array}$


Human epidermal growth factor receptor】HER-2】

\begin{tabular}{llllll}
\hline negative & 20 & 6 & 30.00 & 14 & 70.00 \\
\hline positive & 22 & 2 & 9.09 & 20 & 90.91
\end{tabular}

a: Chi square test, $P<0.05$.

\section{Figures}

HE Staining

ATM
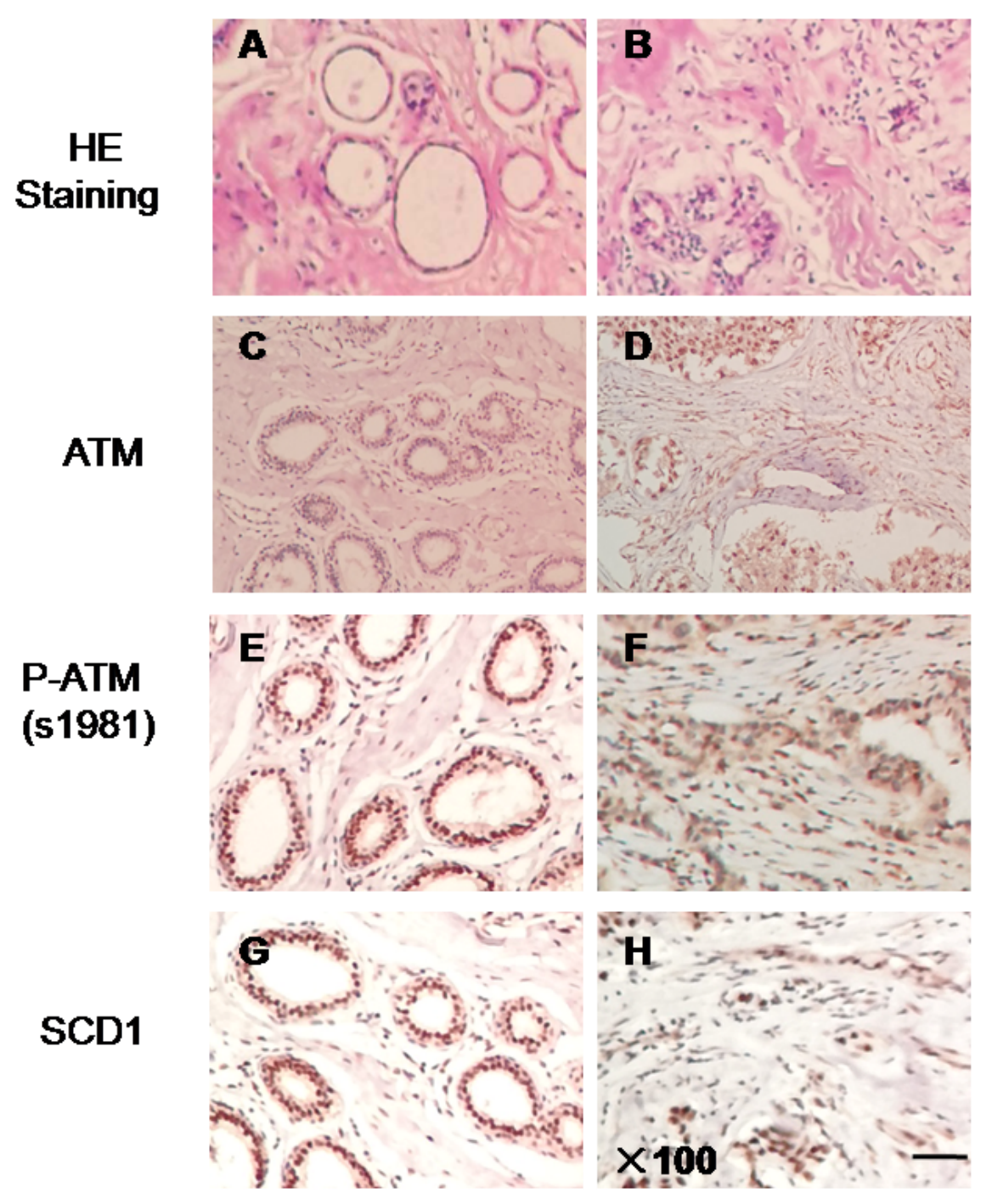

Paracancer tissue Breast cancer tissue

Figure 1 
The protein expression levels of ATM, P-ATM(s1981) and SCD1 in breast cancer tissues and breast paracancer tissues were detected by HE or IHC staining. A and B. Tissues were stained by HE. C and D. The protein expression of ATM in breast cancer specimens was analyzed by IHC staining with the indicated antibody. E and F. The protein expression of p-ATM (s1981) in breast cancer specimens was detected by IHC staining with the indicated antibody. $\mathrm{G}$ and $\mathrm{H}$. The protein expression of SCD1 in breast cancer tissues was determined by IHC staining with the indicated antibody. Magnification of all pictures, $\times 100$; Scale bar, $50 \mu \mathrm{M}$.

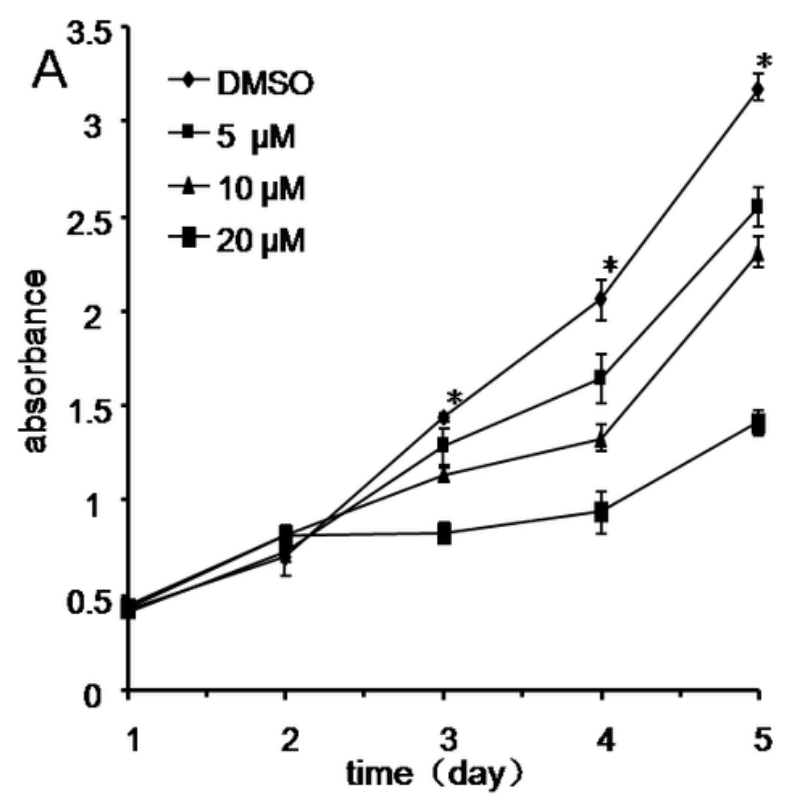

B

Fig 2
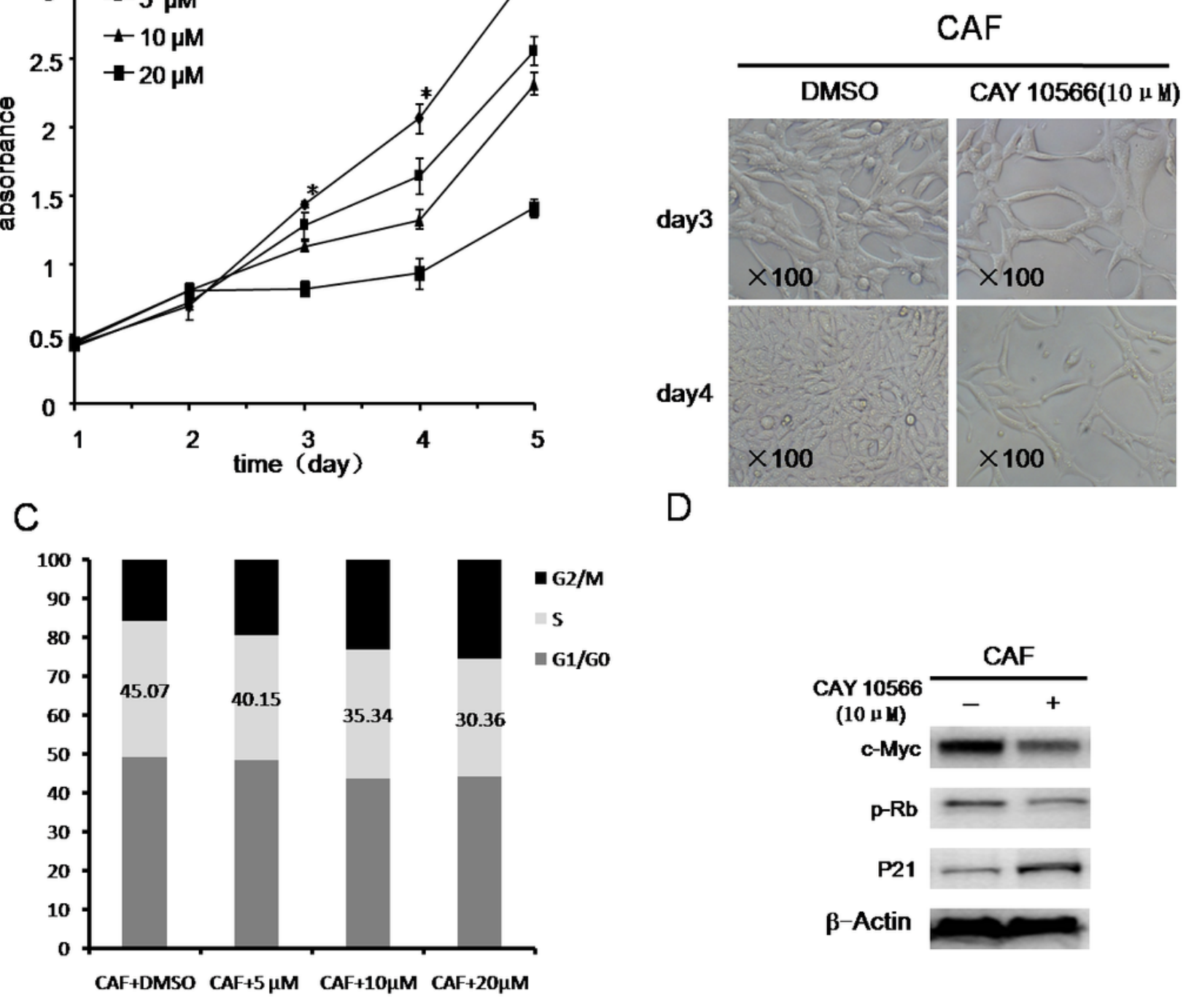

D

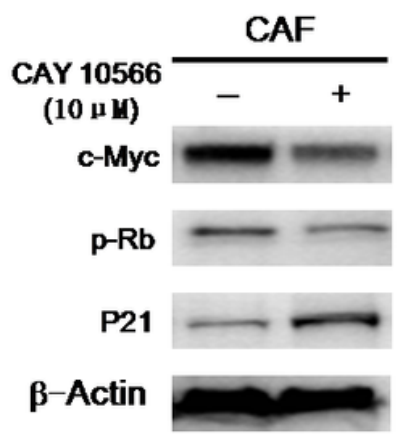

Figure 2

SCD1 promotes the proliferation of breast CAF A. Growth curves of CAF under treatment with or without different doses of CAY 10566 were analyzed with MTT assay for 5 days. $\left({ }^{*}, P<0.05\right)$. B. Photomicrographs of CAF in the presence or absence of CAY10566 $(10 \mu \mathrm{M})$ for 3 days or 4 days. C. Flow cytometry analyses of CAF with or without different doses of CAY 10566 for $24 \mathrm{~h}$. D. CAF was treated with or without CAY10566 $(10 \mu \mathrm{M})$ for $24 \mathrm{~h}$. Immunoblotting analyses were performed with the indicated antibodies. 


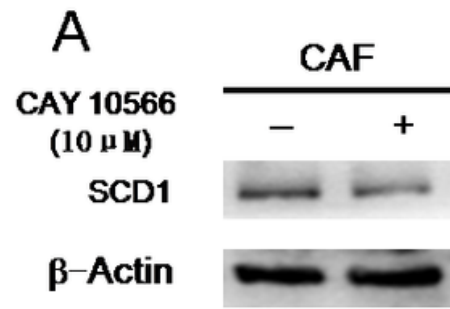

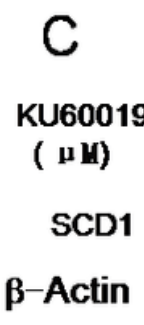
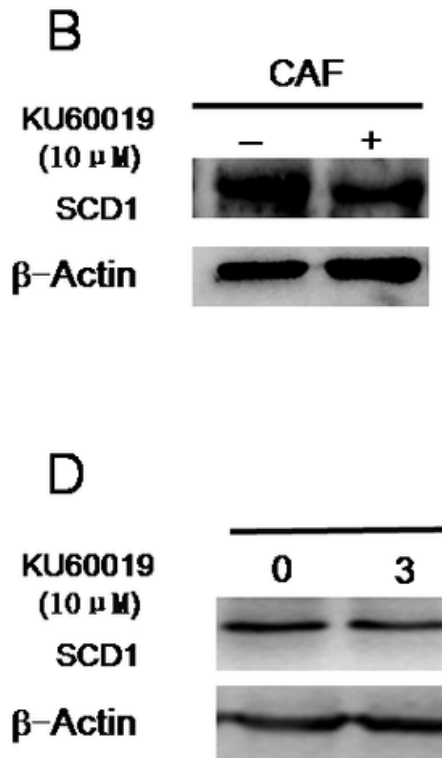

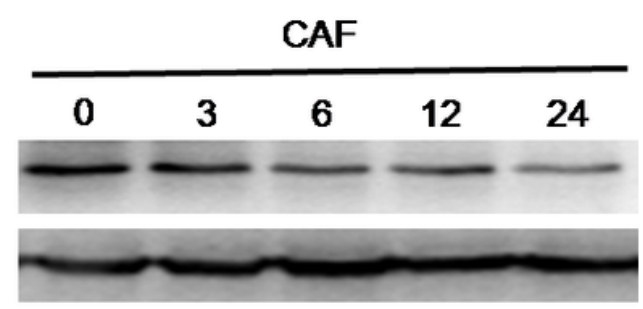

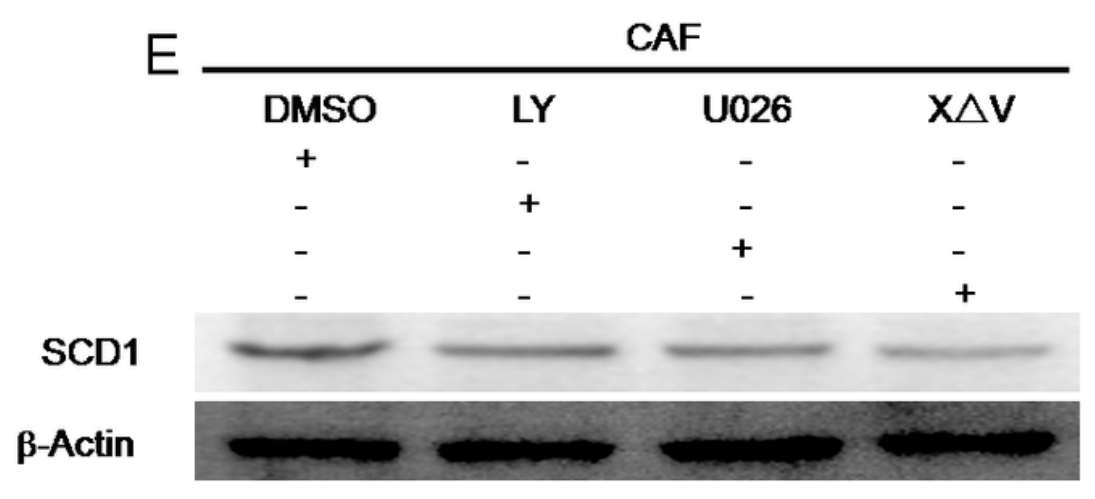

Figure 3

Oxidative ATM protein kinase promotes the proliferation of breast cancer CAF via upregulating the protein expression of SCD1 A. CAF was treated without or with CAY10566 $(10 \mu \mathrm{M})$ for $24 \mathrm{~h}$. Immunoblotting analyses were performed with the indicated antibodies. B. CAF was treated without or with KU60019 (10 $\mu \mathrm{M})$ for $24 \mathrm{~h}$. Immunoblotting analyses were performed with the indicated antibodies. C. CAF was treated with different doses of KU60019 for $24 \mathrm{~h}$. Immunoblotting analyses were performed with the indicated antibodies. D. CAF was treated with KU60019 $(10 \mu \mathrm{M})$ for the designed time. Immunoblotting analyses were performed with the indicated antibodies. E. CAF was treated separately with LY294002 $(20 \mu \mathrm{M})$, U0126 $(25 \mu \mathrm{M})$, and XAV939 $(10 \mu \mathrm{M})$ for $24 \mathrm{~h}$. Immunoblotting analysis was done with the indicated antibodies.

\section{Supplementary Files}


This is a list of supplementary files associated with this preprint. Click to download.

- supplementaryfig1.tiff 